\title{
Arreglo de Microelectrodos Planares con Procesos CMOS Estándar (Semiconductor Complementario Metal-Oxido)
}

\author{
Francisco López ${ }^{(1)}$, Blanca S. Soto ${ }^{(2)}$, Carlos Zúñiga $^{(3)}$, y Salvador Alcántara ${ }^{(2)}$ \\ (1) Facultad de Ciencias Físico Matemáticas, Benemérita Universidad Autónoma de Puebla, \\ Av. San Claudio y 18 Sur, Col. San Manuel, C. U. 72570, Puebla-México. \\ (e-mail: flo012579@gmail.com) \\ (2) Centro de Investigación en Dispositivos Semiconductores, Benemérita Universidad Autónoma \\ de Puebla, Boulevard Valsequillo y 14 Sur, Col. San Manuel, C. U. 72570, Puebla-México. \\ (3) Instituto Nacional de Astrofísica Óptica y Electrónica. Tonantzintla, 72840, Puebla-México.
}

Recibido Sep. 30, 2010; Aceptado Oct. 22, 2010; Versión Final recibida Nov. 29, 2010

\begin{abstract}
Resumen
Se presenta el diseño e integración en el mismo substrato, de un arreglo de microelectrodos planares y el circuito de lectura implementado en un proceso comercial estándar CMOS (Semiconductor Complementario Metal-Oxido), de $0.6 \mu \mathrm{m}$. El diseño incluye el blindaje alrededor del arreglo de microelectrodos y el control del filtro pasabanda a través de la compuerta de transmisión CMOS operando en la región de subumbral. Esto se logra variando el voltaje de compuerta en un rango de 400 a $800 \mathrm{mV}$ para un rango de frecuencia de corte bajo de 1 hasta 1 $\mathrm{KHz}$. El funcionamiento del circuito con polarización de $\pm 1.5 \mathrm{~V}$ dio una ganancia de $40 \mathrm{~dB}, \mathrm{PSRR}$ (razón de rechazo de la potencia aplicada) de $44 \mathrm{~dB}$ y CMRR (razón de rechazo de señales comunes) de $87 \mathrm{~dB}$ en un área $0.014 \mathrm{~mm}^{2}$ haciéndolo un buen prospecto para la aplicación biológica.
\end{abstract}

Palabras clave: CMOS; microelectrodo; compuerta de transmisión; bipotenciales; filtro pasabanda.

\section{Planar Microelectrode Array with CMOS Standar Process (Complementary Metal-Oxide-Semiconductor)}

\begin{abstract}
The design and the on-chip integration of planar microelectronic array and the read-out circuit implemented by $0.6 \mu \mathrm{m}$ CMOS process (Complementary Metal-Oxide-Semiconductor), is presented. The design includes a shielding ring around the microelectrode array and a control for a tunable low pass filter, which are made with a CMOS transmission gate operating in subthreshold region. This is achieved by varying the gate voltage in a range from 400 to $800 \mathrm{mV}$ for a frequency range of 1 to $1 \mathrm{KHz}$. The performance of the circuit with a voltage supply $\pm 1.5 \mathrm{~V}$ was $40 \mathrm{~dB}$ gain, 44 $\mathrm{dB}$ PSRR (power supply rejection ratio) and $87 \mathrm{~dB}$ CMRR (common-mode rejection ratio), in an area of $0.014 \mathrm{~mm}^{2}$, showing that the system is a good alternative for biological applications.
\end{abstract}

Keywords: CMOS; microelectrode; transmission gate, biopotentials; passband filter 


\section{INTRODUCCIÓN}

Desde hace algunas décadas existe información sobre los potenciales eléctricos, éstos generalmente se obtienen a través de las membranas que envuelven las células vivas, como pueden ser neuronas, nervios, músculos y glándulas. De la misma forma, existen diversas células de las plantas que también exhiben este tipo de biopotenciales (Neher, 1992. Geisinger, 2005. Winnie et al., 2006. El cuaderno de por qué biotecnología [en línea]). Para medir estos biopotenciales, se han creado sistemas de registro, los cuales utilizan métodos electrofisiológicos ya sea a través de microelectrodos intracelulares o de extracelulares, los cuales a su vez pueden ser invasivos o no invasivos. Estos sistemas de registro pueden operar, bajo condiciones de estímulo o sin él tanto para células crecidas in vitro o in vivo. Un ejemplo de este tipo de sistemas es el caso del espectro de EEG (electroencefalograma), el cual ha servido para vislumbrar las patologías intracranianas (Suerias y Garcia, 2009). En neurobiología, existen grandes expectativas por entender la riqueza y complejidad del pensamiento y del comportamiento del ser humano, siendo una de las áreas más apasionantes dentro de la neurociencia el estudio de los mecanismos del comportamiento global de la actividad cerebral. Numerosos han sido los esfuerzos para identificar y analizar este comportamiento, en décadas pasadas, los sistemas que registraban los biopotenciales neuronales tenían el problema de baja resolución espacial debido a los problemas tecnológicos en el control de las dimensiones de cada sitio de registro y del propio arreglo. Adicionalmente a este problema, las investigaciones sobre las características de organización estructural, capacidad dinámica y respuesta transitoria de la red neuronal en animales como: ratas, monos, gatos, aves, entre otros permanecían inexploradas principalmente porque la información generada de la actividad, reconocimiento, manipulación y almacenaje requiere una respuesta rápida en el acondicionamiento y procesamiento de la señal registrada (Song et al., 1998. Narayanan, 2004. Wattanapanitch et al., 2007). El mejor desarrollo en el registro de la actividad intracelular se obtuvo cuando un microelectrodo de vidrio o pipeta perforaba el tejido para medir la actividad de la célula. Sin embargo, este método tuvo limitaciones tecnológicas y metodológicas como por ejemplo, la imposibilidad técnica de conectar simultáneamente más de dos sitios (microelectrodos intracelulares), impidiendo el registro intracelular de 5-10 neuronas (Webster, 2009), o de mantener los registros por largo tiempo. Debido a estas limitaciones y al creciente interés en profundizar el estudio de la actividad de la red neuronal, el objetivo en estos últimos 20 años ha sido el desarrollo de técnicas de registro no invasivas utilizando métodos ópticos o técnicas de microelectrodos de película delgada, las cuales siguen en constante evolución (Gross y Lucas 1982. Berdondini et al., 2006). Este último hace uso de microelectrodos de metal noble como son platino negro $u$ oro por tener propiedades adecuadas para el registro (Van et al., 2004). Sin embargo, la interfaz metal/electrolito presenta una muy alta impedancia, lo que provoca que la señal registrada sufra una atenuación o disminución en amplitud. Por otro lado, la poca información en la literatura sobre la integración de los microelectrodos con los circuitos electrónicos presenta limitaciones técnicas o tecnológicas. Por ejemplo, existen sistemas de registro neuronal del tipo invasivos e implantables (con punta de platino negro), los cuales se insertan directamente en la masa encefálica, pero cuentan con un número limitado de microelectrodos (Najafi et al., 1990. Wassum et al., 2008). Por estas razones, los sistemas de registro de biopotenciales extracelulares compuestos de varios microelectrodos metálicos y los instrumentos de acondicionamiento se han investigado por caminos diferentes.

Considerando los inconvenientes anteriores, es necesario realizar estudios relacionados con sistemas de registro de biopotenciales que mejoren la comunicación con las neuronas y de manera simultánea incrementen el tiempo de registro. Los microelectrodos basados en silicio cristalino presentan una menor impedancia y una alta compatibilidad con las neuronas (Soto et al., 2006). Por esta razón, en este trabajo proponemos la integración del arreglo de microelectrodos con los circuitos de lectura en el mismo sustrato, utilizando el proceso comercial estándar metal oxido semiconductor complementario (CMOS) de $0.6 \mu \mathrm{m}$, con lo cual se tendrá una alta reproducibilidad y control en las dimensiones de los dispositivos fabricados. Esto nos permitirá obtener información de la actividad celular de los biopotenciales extracelulares que nos permita investigar sobre el comportamiento de las células neuronales. 
En las subsecuentes secciones se presenta en el marco teórico la electrónica de lectura y acoplada en DC, con lo cual se proporciona una detección simultánea en los sitios de registro. En la sección 2 se reportan los requerimientos mínimos del circuito de lectura y los microelectrodos, los parámetros eléctricos necesarios para su desarrollo. En la sección 3 se describe el diseño del amplificador y del filtro para el registro de la actividad neuronal. En la sección 4 se muestran los resultados obtenidos en la caracterización del funcionamiento eléctrico tanto del circuito de lectura como de la integración final del arreglo de microelectrodos. Finalmente, en la sección 5 se dan las conclusiones derivadas de este trabajo y se plantea el trabajo futuro.

\section{MATERIALES Y MÉTODO}

La topología completa para el registro de la actividad neuronal incluye un amplificador, un filtro pasabanda y la zona de registro (microelectrodo). Los microelectrodos se diseñaron e integraron en un sustrato tipo $\mathrm{P}$, el cual tiene dimensiones de $2.5 \mathrm{~mm}$ por lado, teniéndose un cuadrado de 1 $\mathrm{mm}$ de lado para la ubicación del arreglo de microelectrodos de $4 * 3$. Los microelectrodos fueron implementados con el proceso comercial CMOS $0.6 \mu \mathrm{m}$ de la tecnología ON Semiconductor, la cual cuenta con dos niveles de polisicilio, tres niveles de metal (aluminio), una capa de alta resistividad y una capa pasivante. El arreglo está dispuesto de $4 * 3$ microelectrodos con dimensiones de $100 \mu \mathrm{m}$ por lado separados por la misma distancia, como se muestra en la Figura 1. El arreglo de microelectrodos está dentro un pozo tipo $\mathrm{N}$ y tiene un anillo de guarda para evitar fugas de corriente y aislar el substrato. La zona de exposición del microelectrodo por el cual se realizará el registro, está hecha con una difusión tipo $\mathrm{P}^{++}$altamente impurificada cuya concentración es aproximadamente $5 e^{16} \mathrm{~cm}^{-3}$. Esta última cuenta con una abertura en el pasivante para exponer el microelectrodo al medio físico.

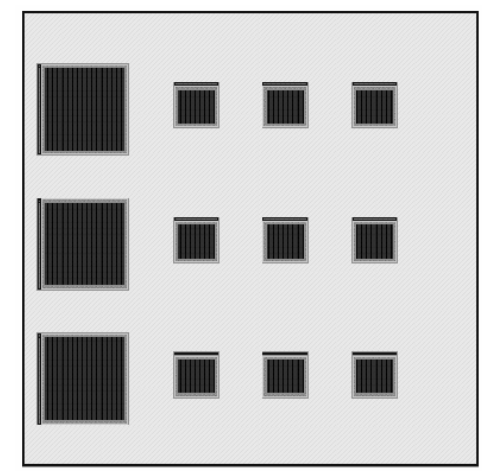

Fig. 1. Arreglo de microelectrodos.

En la Figura 2 se presenta el circuito de lectura diseñado para el registro de la actividad neuronal. Esta implementación se usa en aplicaciones de señales biológicas en donde el ancho de banda es de 1-100 Hz. Para determinar la frecuencia de corte bajo se utiliza la relación (1), en donde la resistencia $R_{\text {mos }}$ se emulara por medio de la compuerta de transmisión trabajando en la región de subumbral (Olsson et al., 2005).

$$
f_{L C}=\frac{1}{2 \pi R_{\text {mos }} C_{2}}
$$

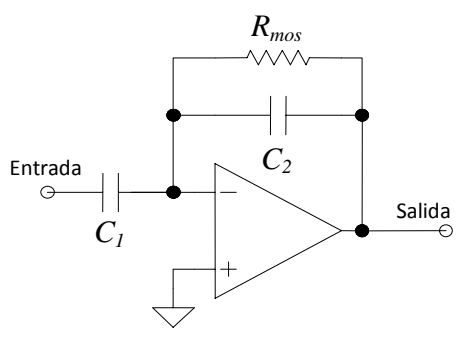

Fig. 2. Circuito empleado para filtrar señales biológicas. 
donde $f_{L C}$ es la frecuencia de corte bajo, $C_{1}$ y $C_{2}$ son capacitores de 10 y $0.1 \mathrm{pF}$ respectivamente, y el valor mínimo del resistor debe ser $R_{\text {mos }} \approx 1.5 \mathrm{G} \Omega$. La simulación de la resistencia se realiza por medio de la compuerta de transmisión CMOS Figura 3. La compuerta de transmisión es ampliamente usada en el diseño de filtros sintonizables debido a que la variación del voltaje de control de la compuerta que pasa por cada transistor provoca un incremento significativo en la resistencia (Uyemura 2002. Bikumandla et al., 2004).

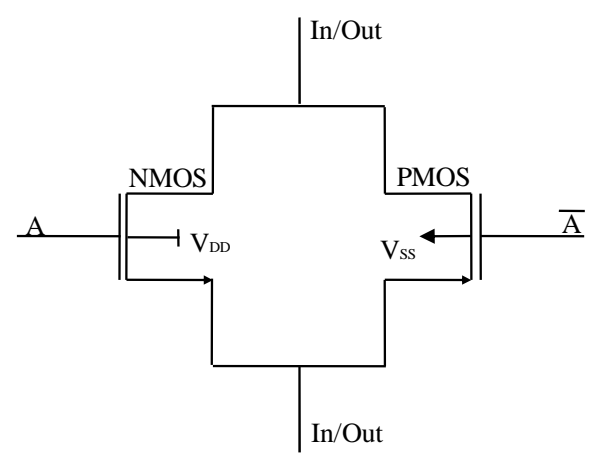

Fig. 3. Compuerta de transmision.

Para determinar el funcionamiento de la compuerta de transmisión, se determinó primero el funcionamiento del MOSFET como interruptor, para ello se empleo el esquemático de la Figura 4, donde se polariza en DC al transistor para observar su comportamiento.

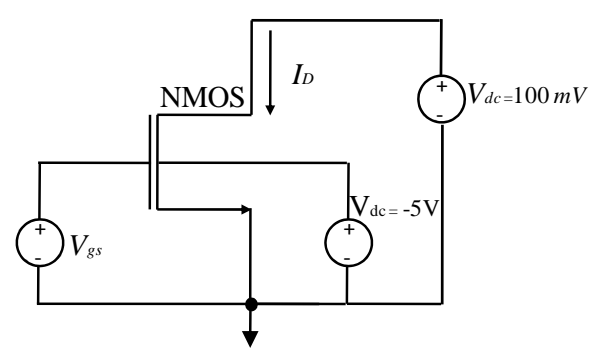

Fig. 4. Interruptor NMOS.

De acuerdo a los resultados obtenidos del uso de los transistores MOS como resistencia se puede obtener una resistencia equivalente de la compuerta de transmisión $R_{C T}$, la cual es definida como:

$R_{C T}=\frac{V_{C T}}{I_{D N}+I_{D P}}$

donde $V_{C T}$ es el voltaje de la compuerta de transmisión, $I_{D N}, I_{D P}$ son las corrientes del drenaje para el transistor NMOS y PMOS, respectivamente.

$I_{D N}=\mu_{N} C_{o x} \frac{W}{L}\left(V_{G S}-V_{T}\right) V_{D S}$

En donde $\mu_{N}$ es la movilidad de los electrones, $C_{o x}$ es la capacitancia del óxido, $W / L$ es la razón de aspecto, $V_{G S}$ es el voltaje de compuerta a fuente, $V_{T}$ es el voltaje de umbral, $V_{D S}$ es el voltaje de drenaje a fuente. La corriente para el transistor PMOS es semejante a la ecuación 3. Para calcular la resistencia $R_{C T}$ se tienen que conocer los valores de las resistencias de los transistores $\mathrm{N}$ y $\mathrm{P}$. Debido a que los transistores se encuentran en paralelo, la resistencia puede escribir como $R_{C T}=$ $R_{N} \| R_{P}$, donde el valor de la resistencia está controlado por la razón de aspecto de los transistores PMOS y NMOS. 


$$
\begin{aligned}
& R_{N}=\frac{1}{\mu_{N} C_{o x} \frac{W}{L}\left(V_{G S}-V_{T}\right)} \\
& R_{P}=\frac{1}{\mu_{P} C_{o x} \frac{W}{L}\left(V_{G S}-V_{T}\right)}
\end{aligned}
$$

Para la integración del arreglo de microelectrodos se empleo el editor de patrones geométricos Virtuoso de $C A D E N C E^{\circledR}$, para la circuito de lectura se empleó un amplificador analógico y un filtro sintonizable (ver Figura 5). El circuito de lectura tiene una ganancia en voltaje de 100. La primera etapa está formada por el par diferencial M1/M2, con la polarización a través de los transistores M5 y M6. El espejo de corriente está formado por M3 y M4. Para mejorar la estabilidad del circuito la compensación se implemento a través del seguidor de voltaje dado por los transistores M7, M9 y el capacitor $C_{1}$. Los transistores M10 y M11 forman el buffer que proporciona un buen manejo de la carga a la salida. Los transistores M12, M13, M14 y M1 forman la compuerta de transmisión usada para formar el filtro y el capacitor $C_{2}$. Las razones de aspecto de los transistores se muestran en la Tabla 1.

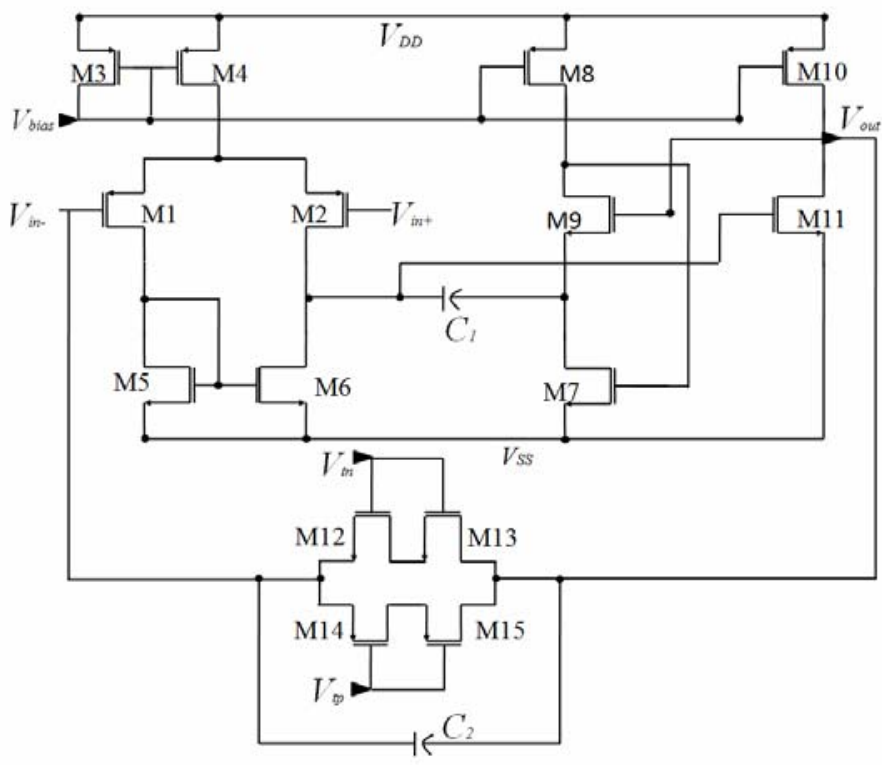

Fig. 5. Esquemático del filtro usado para señales biológicas.

Tabla 1. Razones de aspecto.

\begin{tabular}{|l|l|l|l|l|l|l|l|l|l|l|}
\hline Tipo & P & P & N & P & P & N & P & N & N & P \\
\hline & $\begin{array}{l}\text { M1- } \\
\text { M2 }\end{array}$ & $\begin{array}{l}\text { M3- } \\
\text { M4 }\end{array}$ & $\begin{array}{l}\text { M5- } \\
\text { M6 }\end{array}$ & M7 & M8 & M9 & M10 & $\begin{array}{l}\text { M1 } \\
1\end{array}$ & $\begin{array}{l}\text { M12- } \\
13\end{array}$ & $\begin{array}{l}\text { M14- } \\
15\end{array}$ \\
\hline $\begin{array}{l}\text { W/L } \\
(\mu \mathrm{m})\end{array}$ & $\begin{array}{l}60 / 4 . \\
8\end{array}$ & $\begin{array}{l}40.0 \\
5 / 2.4\end{array}$ & $\begin{array}{l}19.95 / \\
5.4\end{array}$ & $\begin{array}{l}10.05 / \\
3\end{array}$ & $\begin{array}{l}40.05 / \\
2.4\end{array}$ & $\begin{array}{l}40.05 / \\
1.5\end{array}$ & $\begin{array}{l}150 / \\
2.4\end{array}$ & $\begin{array}{l}75 / \\
2.4\end{array}$ & $\begin{array}{l}10.05 / \\
1.5\end{array}$ & $\begin{array}{l}10.05 / \\
1.5\end{array}$ \\
\hline
\end{tabular}

Los patrones geométricos (layouts) fueron realizados utilizando las dimensiones mínimas permitidas por las reglas de diseño de la tecnología, lo cual permite reducir en gran medida el consumo de potencia y las capacitancias parásitas en todos los nodos críticos. Los elementos 
parásitos de los circuitos fueron extraídos mediante la herramienta Virtuoso Extracted que contiene la información de las capas disponibles en la tecnología para formar los dispositivos electrónicos. Por todo el Layout se comprueba información y como resultado se identifican los dispositivos diseñados y los elementos parásitos asociados a ellos. En la Figura 6, se muestra un transistor con capacitancias parasitas asociadas, los cuales se obtuvieron utilizando los parámetros tecnológicos del proceso CMOS $0.6 \mu \mathrm{m}$ de la tecnología ON Semiconductor [en línea]. En la Figura 7 se muestran el Layout del amplificador operacional, aplicando la técnica Serpentín. El área del circuito está conformada por las áreas de los transistores $\mathrm{P}$ y $\mathrm{N}$, así como las líneas de entrada, salida y alimentación. Los resultados eléctricos obtenidos de las simulaciones son derivados del análisis realizado con el simulador de circuitos spectres. Este entorno realiza simulaciones de rendimiento y comportamiento a nivel de transistores, aproximándose cada vez más a su comportamiento real, una vez realizado el circuito en fábrica. La exactitud de la simulación dependerá del modelado de los transistores, que para nuestro caso se centra en los modelos BSIM3v3.1 (Liu et al., 2003). Además, este simulador efectúa el cálculo de todos los efectos de segundo orden, como por ejemplo: saturación de la velocidad, degradación de la movilidad, modulación de la longitud de canal, efecto del cuerpo, entre otros (Liu et al., 2003. Benumof et al., 1998).

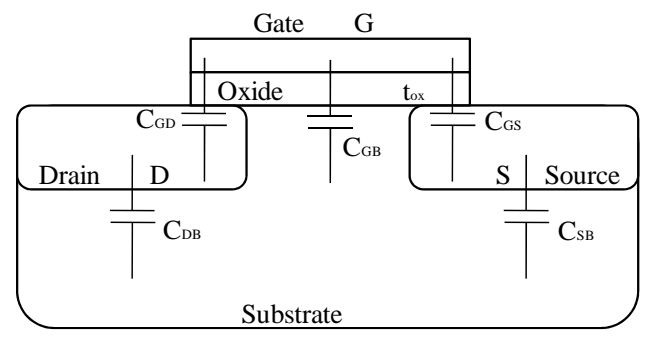

Fig. 6. Transistor MOS con capacitancias parásitas.

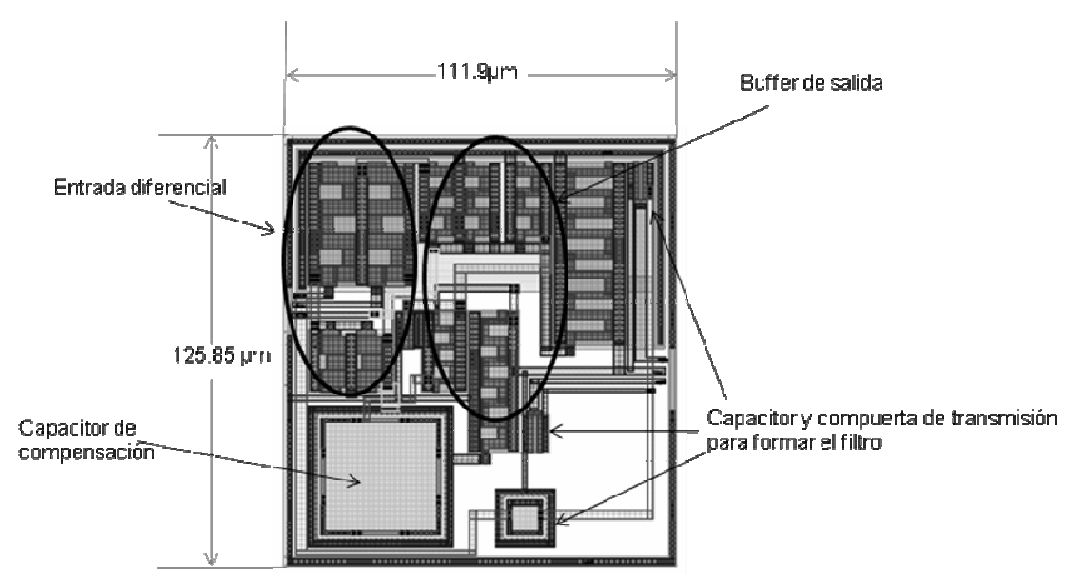

Fig. 7. Layout del amplificador y filtro para el registro de la actividad neuronal.

\section{RESULTADOS Y DISCUSIÓN}

El circuito de lectura y el arreglo de microelectrodos para el registro de la actividad neuronal fueron implementados en el proceso tecnológico CMOS $0.6 \mu \mathrm{m}$ de la tecnología ON Semiconductor. La Figura 8 muestra el Layout final del arreglo de microelectrodos y los circuitos de lectura, los cuales fueron distribuidos de la siguiente manera: $3 * 3$ microelectrodos cuyas dimensiones son de $100 \mu \mathrm{m}$ de lado, y un arreglo de $1 * 3$ de $200 \mu \mathrm{m}$ por lado. Esta distribución nos permitió concentrar el arreglo completo en un 16\% del área del chip. Por otro lado, el circuito de lectura que consta del amplificador y el filtro, se diseño con la técnica de Layout Serpentín debido principalmente a que se minimizan las áreas de fuente y drenaje, reduciendo las capacitancias parásitas de interconexión y las capacitancias parásitas entre dispositivos por al menos $67 \%$. El circuito de lectura tiene una ganancia de aproximadamente $40 \mathrm{~dB}$, una razón de rechazo en modo 
común (CMRR) de $87 \mathrm{~dB}$, una relación de rechazo a la fuente de alimentación de $44 \mathrm{~dB}$ y un voltaje de offset de $100 \mu \mathrm{V}$, este valor es grande debido que se aplico la técnica Serpentín, pero se obtiene una mayor integración de microelectrodos con los circuitos de lectura. El circuito de lectura está alimentado con $\pm 1.5 \mathrm{~V}$, presenta una banda sintonizable de 1 hasta $1 \mathrm{KHz}$ en la frecuencia de corte bajo. Los resultados obtenidos en frecuencia para el filtro pasabanda se muestran en la Figura 9, donde se observa la sintonización de la frecuencia de corte baja al variar el voltaje de control, $V_{t n}$ de $400-700 \mathrm{mV}$, mientras que la frecuencia de corte alto se mantiene constante. La Figura 10 muestra un CMRR de $87 \mathrm{~dB}$, con lo cual se garantiza un rechazo al ruido con una exactitud del 10\% en un rango de frecuencias de 100 a $20 \mathrm{KHz}$. La Figura 11 muestra la relación al rechazo a las fuentes de alimentación, donde se obtuvo un valor de $44 \mathrm{~dB}$ en el rango de frecuencias de 100 a $20 \mathrm{KHz}$. Las impedancias de entrada y salida obtenidas son de $350 \mathrm{M} \Omega$ y $990 \Omega$. El ruido del filtro es de $1.56 \mu \mathrm{V} / \sqrt{ } \mathrm{Hz}$, el cual es menor que la señal de la neurona, con la cual no se ve afectada la lectura final de la actividad. En la Tabla 2 se muestran un resumen de los resultados de las simulaciones eléctricas para el circuito de lectura.

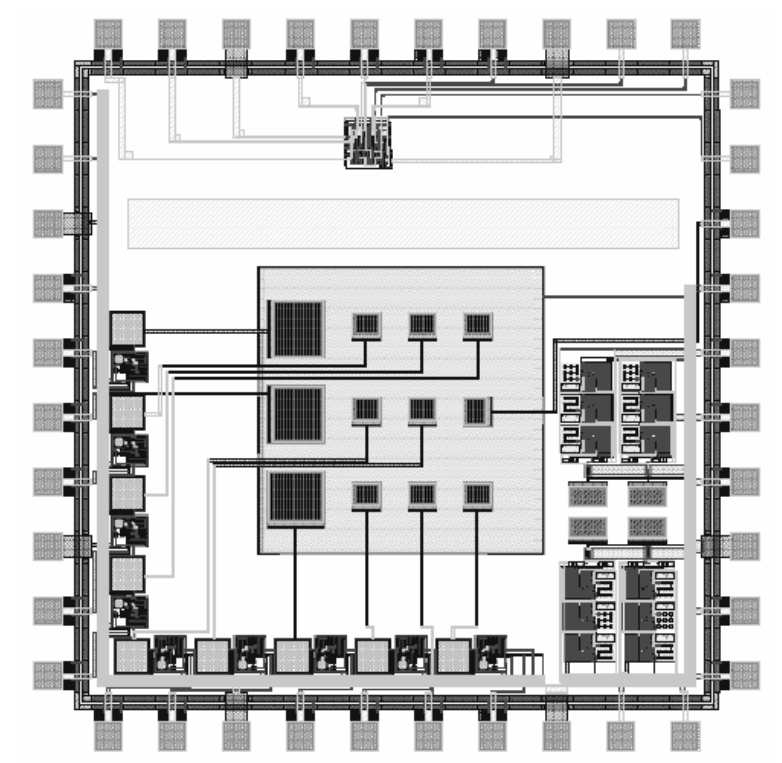

Fig. 8. Layout final de la integración de los microelectrodos con los circuitos de lectura.

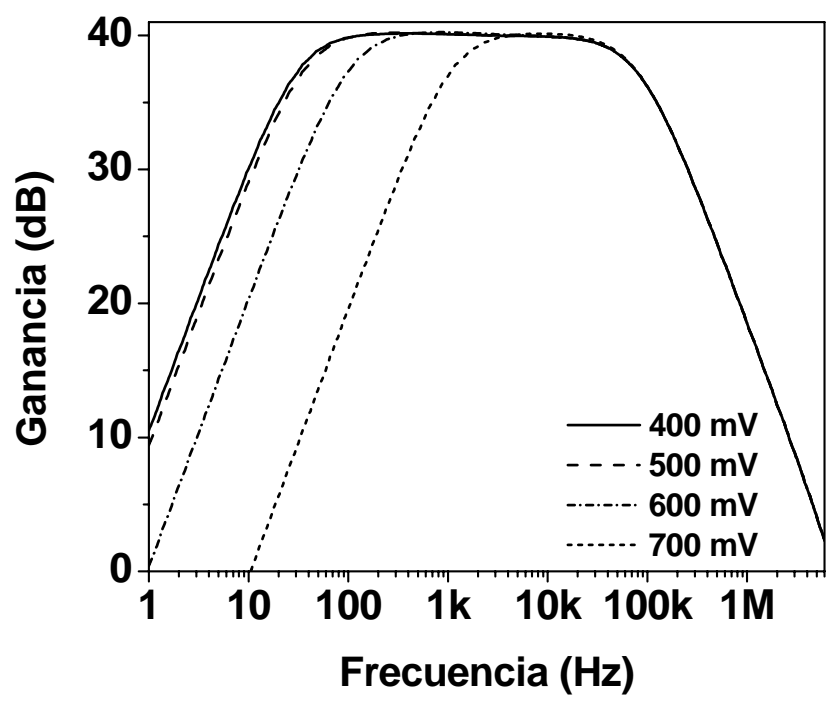

Fig. 9. Sintonización en la frecuencia de corte bajo al variar el voltaje $V_{t n}$. 


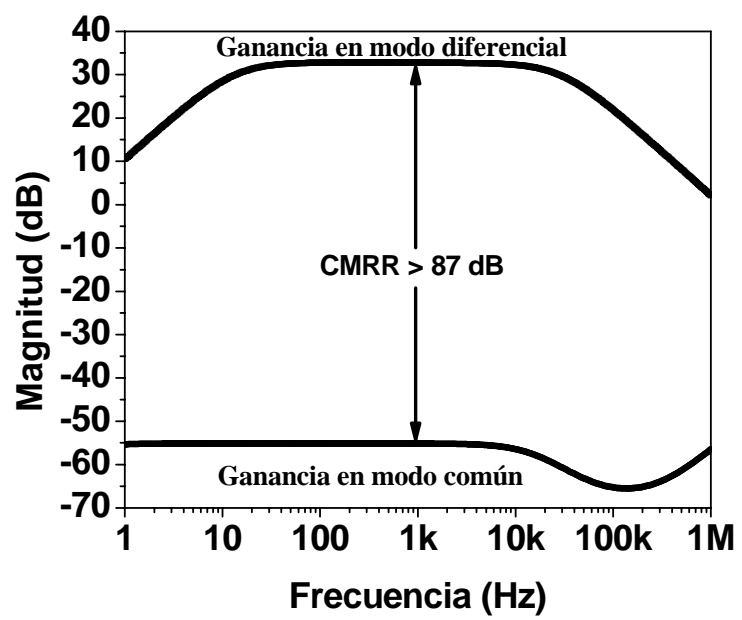

Fig. 10. CMRR del circuito de lectura usada para registrar la actividad neuronal.

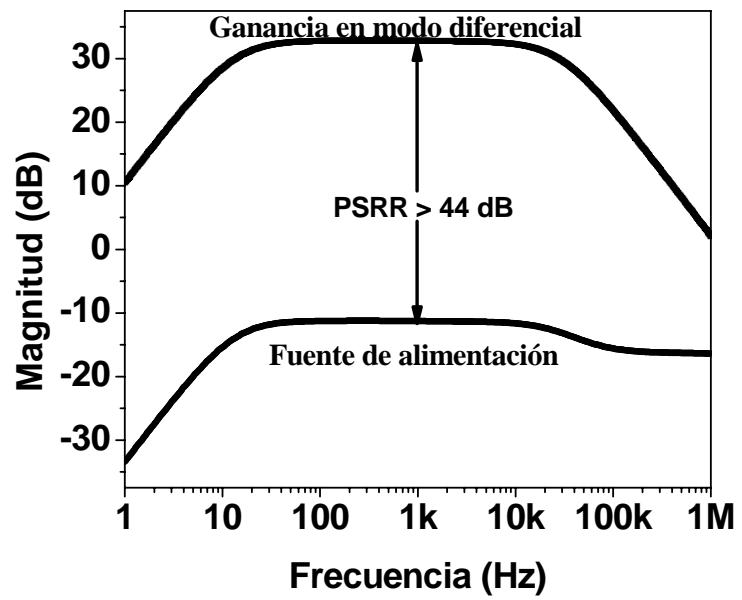

Fig. 11. PSRR del circuito de lectura usada para registrar la actividad neuronal.

Tabla 2. Especificaciones Eléctricas Obtenidas.

\begin{tabular}{|l|l|}
\hline Parámetros & Filtro \\
\hline Área $\left(\mathrm{mm}^{2}\right)$ & .014 \\
\hline Ganancia $(\mathrm{dB})$ & 40 \\
\hline CMRR $(\mathrm{dB})$ & 87 \\
\hline PSRR $(\mathrm{dB})$ & 44 \\
\hline Alimentación (Volts) & \pm 1.5 \\
\hline Offset $(\mu \mathrm{V})$ & 100 \\
\hline
\end{tabular}




\section{CONCLUSIONES}

Presentamos la integración del arreglo de microelectrodos $4 * 3$ con los circuitos de lectura en el proceso CMOS estándar de $0.6 \mu \mathrm{m}$.

Para el circuito de lectura analógica se logró un filtro pasabanda sintonizable en la frecuencia de corte baja de $1 \mathrm{~Hz}$ a $1 \mathrm{KHz}$, al variar el voltaje aplicado en la compuerta de transmisión en un rango de $400-700 \mathrm{mV}$. Las características obtenidas cumplieron con las especificaciones de diseño: ganancia de $40 \mathrm{~dB}$, PSRR de $44 \mathrm{~dB}$, CMRR de $87 \mathrm{~dB}$, corriente de polarización de $60 \mu \mathrm{A}$, offset $100 \mu \mathrm{V}$ y un área de $0.014 \mathrm{~mm}^{2}$. Comparando estos resultados con los reportados en el trabajo de Woradorn y cols., en 2005, se logro un voltaje de alimentación 53\% menor. Respecto al un consumo de área se disminuyo un 87\%, un CMRR 30\% mayor al optimizar el Layout con la técnica Serpentín debido principalmente a que se minimizan las áreas de fuente y drenaje. El sistema propuesto en este trabajo no emplea el bloque adicional de buffer o filtro de salida, ya que éste se implementa directamente en el amplificador operacional y sustituye la función de éste reduciendo considerablemente el consumo de área.

Finalmente, la figura de mérito obtenida se encuentra dentro de las recomendaciones generales, en cuanto a cercanía entre el sitio de detección y la electrónica de lectura, área reducida y bajo consumo de potencia. Además de que la propuesta es viable para la integración de MEMS con el arreglo de microelectrodos planares basados en silicio sobre un mismo substrato para el registro de la actividad neuronal.

Como trabajo futuro se plantea el postproceso del circuito integrado fabricado y la caracterización eléctrica del mismo.

\section{AGRADECIMIENTOS}

Los autores agradecen el apoyo económico a través del Programa de Fortalecimiento al Posgrado Nacional de CONACYT, al Instituto de Ciencias de la BUAP y al Profesor Agustín Leobardo Herrera May de la Universidad Veracruzana por sus valiosos comentarios y sugerencias.

\section{REFERENCIAS}

Benumof R, Zoutendyk J, Coss J. Models of second-order effects in metal-oxide-semiconductor field-effect transistors for computer applications, Journal of Applied Physics, 63, 5131-5142 (1998).

Berdondini L, Chiappalone M, Van der Wal P, Imfeld K, Rooij N, Koudelka-Hep M, Tedesco M, Martionia S, Van J, Masson G, Garenne A. A microelectrode array (MEA) integrated with clustering structures for investigating in vitro neurodynamics in confined interconnected sub-populations of neurons, Sensors and Actuators B: Chemical, 114(1), 530-541 (2006).

Bikumandla M, Ramírez J, Urquidi C, Carvajal R, López A, Biasing CMOS amplifiers using MOS transistors in subthreshold region, IEICE Electronic Express 1(12), 339-345 (2004).

El cuaderno de por qué biotecnología: http://www.porquebiotecnologia.com.ar Acceso: 27 Junio (2010).

Geisinger D, Electrodos Implantables en el Cerebro en Aplicaciones de Prótesis Neuronales, XIV Seminario de Ingeniería Biomédica, 1-6, Uruguay (2005).

Gross G, Lucas J, Long-term monitoring of spontaneous single unit activity from neuronal monolayer networks cultured on photoetched multielectrode Surfaces, J. Electrophys. Tech., 9, 5569 (1982).

Liu W, Jin X, Xi X, Chen J, Jeng M, Liu Z, Cheng Y, Chen K, Chan M, Hui K, Huang J, Tu R, Ko K, Hu C, BSIM3v3.1 MOSFET Model. Berkeley (2003).

Najafi K, Ji J, Wise K, Scaling limitations of silicon multichannel recording probes, IEEE Trans. Biomed. Eng., 37(1), 1-11 (1990). 
Narayanan S, Neuronal Development, Lectures Spring, 1-74, Berkeley (2004).

Neher E, Ion channels for communication between and within cells, Science, 256(5056), 498-502 (1992).

Olsson R, Buhl D, Sirota A, Buzsaki G, Wise K, Band-Tunable and Multiplexed Integrated Circuits for simultaneous Recording and Stimulation with Microelectrode Arrays, IEEE Trans. Biomed. Eng., 52(7), 1303-1311 (2005).

On Semiconductor: http://www.onsemi.com Acceso: 30 Abril (2010).

Song H, Ming G, He Z, Lehmann M, McKerracher L, Tessier-Lavigne M, Poo M, Conversional of Neuronal Growth Cone Responses from Repulsion to Attraction by Cyclic Nucleotides, Science, 281(5382), 1515-1518 (1998).

Soto B, Sosa J, López F, Vazquez J, Characterization and properties of alternative materials on electrode microstructures in biomedical applications, III Encuentro Participacion de la mujer en la ciencia, S4-BYQ12 (2006).

Suerias M, García R, El electroencefalograma en urgencias epilépticas, Revista del grupo de epilepsia de la SEN, 3, 12-15 (2009).

Uyemura J, CMOS logic circuit design, $1^{\text {a }}$ edicion, 259-283. Kluwer Academic Publishers, New York, USA (2002).

Van J, Wolters P, Corner M, Rutten W, Ramakers G, Long-Term Characterization of Firing Dynamics of Spontaneous Burst in Cultured Neuronal Networks, IEEE Trans. Biomed. Eng., 51(11), 2051-2062 (2004).

Wassum K, Tolosa V, Wang J, Walker E, Monbouquette H, Maidment N, Silicon Wafer-Based Platinum Microelectrode Array Biosensor for Near Real-Time Measurement of Glutamate in VIVO, Sensors, 8 5023-5036 (2008).

Wattanapanitch W, Fee M, Sarpeshkar R, An Energy- Efficent Micropower Neuronal Recording Amplifier. IEEE Trans. Biomed. Circuit and Syst., 1(2), 136-147 (2007).

Webster J, Medical Instrumentation, Application and Design, $4^{\mathrm{a}}$ edition, John Wiley \& Sons Inc, USA. 215-219 (2009).

Winnie J, Yoshida K, Hofmann U, In-Vivo Implant Mechanics of Flexible, Silicon-Based ACREO Microelectrode Arrays in Rat Cerebral Cortex, IEEE Trans. Biomed. Eng., 53(5), 934-940 (2006). 